\title{
Fast algorithm for 3-D vascular tree modeling
}

\author{
Marek Kretowski ${ }^{\text {a,b }}$, Yan Rolland ${ }^{\text {a,c }}$, Johanne Bézy-Wendling ${ }^{\text {a,*, }}$ \\ Jean-Louis Coatrieux ${ }^{\text {a }}$ \\ a Laboratoire Traitement du Signal et de l'Image, INSERM EMI 9934, Université de Rennes 1, Campus de Beaulieu, \\ 35042 Rennes Cedex, France \\ ${ }^{\mathrm{b}}$ Institute of Computer Science, Technical University of Bialystok, Wiejska 45a, 15-351 Bialystok, Poland \\ c Département de Radiologie et d'Imagerie Médicale, CHR Hôpital Sud, Rennes, France
}

Received 22 June 2001; received in revised form 15 October 2001; accepted 12 November 2001

\begin{abstract}
In this short paper, accelerated three-dimensional computer simulations of vascular trees development, preserving physiological and haemodynamic features, are reported. The new computation schemes deal: (i) with the geometrical optimization of each newly created bifurcation; and (ii) with the recalculation of blood pressures and radii of vessels in the whole tree. A significant decrease of the computation time is obtained by replacing the global optimization by the fast updating algorithm allowing more complex structure to be simulated. A comparison between the new algorithms and the previous one is illustrated through the hepatic arterial tree.
\end{abstract}

(C) 2002 Elsevier Science Ireland Ltd. All rights reserved.

Keywords: Vascular modeling; Fast computing; Optimization

\section{Introduction}

The capability to model living systems is becoming more and more important due to the fast growing knowledge in all areas from cell to function. Modeling provides new ways to better understand complex interactions between elementary mechanisms and behaviors of the whole organ. It often leads to meaningful simulations in which the level of details (or, in other words, the complexity

* Corresponding author. Tel.: + 33-223-235604; fax: + 33223-236917.

E-mail address: johanne.bezy@univ-rennes1.fr (J. BézyWendling). of the models) is controlled. Effective methods for the modeling of anatomically-based structures and physiologically-based processes are required to enable advances in integrative physiology as well as in virtual reality. However, as it is shown here for the vascular system, they must be computationally tractable to be of practical significance.

Several models of the vascular network have been proposed (reader may refer to [1] for a detailed survey). These models allow structural and functional features to be changed and pathological vascular modifications to be rendered. They range from the early 2-D models [2] to more recent surface-based ones [1] or to fully 3-D models $[3,4]$ with different objectives such as realistic 
representations for rendering applications [1], physiological interpretation [3] or organ-based descriptions like coronary arterial trees $[4,5]$. The present contribution is a continuation of the work described in [6]. Here our objective is to significantly reduce the computation time, in order to generate trees with more than 10000 extremities, in a reasonable time. We concentrate on the most critical (i.e. the most time consuming) steps, mainly involved in the network optimization. A brief review of the organ growth features is provided in Section 2 while acceleration cues are detailed in Section 3. An application to the liver arterial tree is displayed in Section 4 and further discussed.

\section{Model description}

The first step to create a realistic model of a vascular tree consists in specifying both the organ 3-D shape and the properties according to which the tree develops. Macro-cells are uniformly distributed inside the organ volume. A macro-cell is an element of tissue characterized by some geometrical (like the size and the spatial position) and haemodynamic properties (the blood flow for instance). Several classes of macro-cells can be defined and can lead to regions having different vascular properties.

The organ growth is modeled as the result of an hyperplasia process (increased number of macrocells). The changes in the number of macro-cells occur at discrete time moments called cycles. In each cycle, a macro-cell has a certain probability $\left(P r_{\mathrm{B}}\right)$ to give birth to a new macro-cell of the same class, or to die $\left(P r_{\mathrm{D}}\right)$. Both probabilities depend on the cell type (class) and the cell age. For instance if healthy and tumoral macro-cells are specified, the pathological ones should have a greater $\operatorname{Pr}_{\mathrm{B}}$ (details about the way initial $P r_{\mathrm{B}}$ and $\operatorname{Pr}_{\mathrm{D}}$ values are chosen and evolve are given in [3]). For each 'mother' macro-cell, a few randomly chosen spatial positions of a 'daughter' cell in the neighborhood are tested against the local constraint of maximal density. A new macro-cell is created only if a proper position is found.

The bounding shape expands periodically, when the corresponding volume is almost filled by macro-cells. An empty space, which appears during the organ growth, is occupied by new macrocells in consecutive cycles. The growth continues until the organ reaches its adult size.

The tree develops to respond to the needs of the expanding tissue. Consequently, the apparition of a new macro-cell leads to the development of a new vessel. Conversely, when a macro-cell disappears, the corresponding vessel retracts and disappears too. Each macro-cell is perfused by exactly one vessel of the vascular tree.

This vascular tree model has a binary structure. It means that interconnections (anastomoses) are not taken into account. Each vessel is represented by a rigid tube and can divide into two other branches creating a bifurcation (Fig. 1). The vessels are defined by their length $(l)$, radius $(R)$ and blood flow rate $(Q)$. Each extremity of the vascular tree is connected to a macro-cell, which is the place of exchange between the tissue and the blood supplied by the vessel.

Two main physical laws have to be taken into account for the design of realistic vascular trees. The law of matter preservation has to be fulfilled at each bifurcation and is given by:

$Q_{\mathrm{b}}=Q_{1}+Q_{\mathrm{r}}$

where $Q_{\mathrm{b}}$ is the blood flow in the parent vessel and $Q_{1}$ and $Q_{\mathrm{r}}$ are blood flows in the left and right daughter branches. It is a rather obvious property: the quantity of blood, which enters a bifurcation, has to leave it.

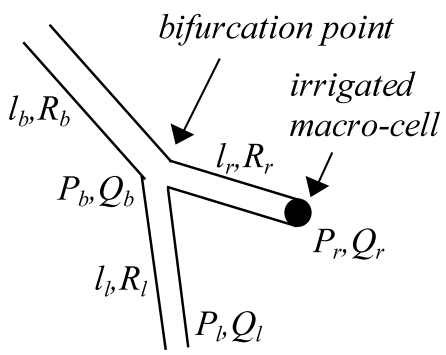

Fig. 1. A bifurcation irrigating a macro-cell. $P_{l}$, pressure at the extremity of the left vessel; $Q_{1}$, blood flow at the extremity of the left vessel; $P_{\mathrm{r}}, Q_{\mathrm{r}}, P_{\mathrm{b}}, Q_{\mathrm{b}}$, corresponding values for the right and the bifurcation vessel. 
In the model, the blood is assumed to be a Newtonian fluid with constant viscosity $(\mu){ }^{1}$ Hence Poiseuille's law is applied to calculate the pressure difference $(\Delta P)$ between the two extremities of a vessel. It depends on the blood flow rate, the length and the radius of the vessel:

$$
\Delta P=Q \frac{8 \mu l}{\pi R^{4}}
$$

Another constraint deals with the decreasing vessel radii in the tree (bifurcation law). It gives the relationship between the radius of the parent vessel $\left(R_{\mathrm{b}}\right)$ and the radii of its two daughter branches $\left(R_{1}\right.$ and $\left.R_{\mathrm{r}}\right)$ :

$R_{\mathrm{b}}^{\gamma}=\alpha R_{\gamma}^{\gamma}+\beta R_{\mathrm{r}}^{\gamma}$.

In [7], $\gamma=6, \alpha=1+Q_{1} / Q_{\mathrm{r}}$ and $\beta=1+Q_{\mathrm{r}} / Q_{1}$ were proposed, which were derived from the minimal volume principle, but in other papers [8-10] $\alpha=\beta=1$ and $\gamma$ varies from 2.55 to 3 . The choice of proper parameters $(\alpha, \beta, \gamma)$ is not an easy task, but the general expression (Eq. (3)) was confirmed both by morphometrical analyses and theoretical studies.

\section{Vascular tree optimization}

It is a widely acceptable fact that the growth and functional behavior of vascular system is governed by some optimality principles, but the definition of the target function which should be minimized (or maximized) is still an open problem (e.g. four different optimality principles were examined in [11]). In the present model, it is assumed that the target function depends only on the radii and lengths of vessels. It is also assumed that the function is additive, and thus summed over all vessels in the tree (for instance the volume of vascular tree can be minimized).

New macro-cells appear and are supplied by the vascular system in a sequential manner. Therefore, they are connected to the vascular tree one by one.

\footnotetext{
${ }^{1}$ In fact the blood has a Newtonian behavior only in vessels whose caliber is higher than $100 \mu \mathrm{m}$, but even for smaller vessels Poiseuille's law can be considered as a good approximation.
}

The geometrical optimization of a bifurcation is separated from the global optimization of the tree. The choice of the bifurcation point is done locally and, with this known geometry, the whole tree is then optimized. It is assumed that the influence of moves of the bifurcation point on the remaining vessels is relatively small and can be neglected during the local optimization. This approximation allows the computational complexity to be significantly reduced leading to the simulation of complex trees in a very short time. Another approach was presented by Karch et al. [4], where all vessel parameters are recomputed during the optimization of a single bifurcation.

\subsection{Geometrical optimization of a single bifurcation}

The perfusion process of a new macro-cell starts by choosing a set of candidate vessels. The distance between all vessels and the macro-cell is calculated and only the closest ones are considered. For each candidate vessel, a new bifurcation is temporarily created and the macro-cell is perfused. Given the spatial positions (coordinates) and the physiological properties of the newly created vessels, a branching point, which minimizes the volume, is searched. To optimize the bifurcation, the 2-D method proposed in [7] was extended to the 3-D case. When the optimal structure is found, pressures and radii of the whole tree have to be recalculated (the global optimization is applied) and then crossing between vessels is checked and candidates are excluded accordingly. In the next step, for each remaining candidate vessel, the volume of the whole tree is computed and the solution corresponding to the minimal value is chosen. The optimal vessel is used to permanently connect the macro-cell to the tree.

\subsection{Optimization of the whole tree}

Assuming that the geometry of the vascular tree is set, the optimized tree (to be determined) must fulfill the following constraints:

- constant pressure $\left(P_{\text {cell }}\right)$ at the extremities, and the blood flow depending on the macro-cell class, 
- Poiseuille's law at each vessel,

- matter preservation and bifurcation law at each bifurcation.

The fully optimized vascular tree is defined as the optimized tree with a given pressure $P_{\mathrm{e}}$ at the entry of the tree.

\subsection{A bifurcation irrigating two cells}

In such a situation, the strategy proposed in [3], for irrigating a new macro-cell, can be used. First $P_{1}=P_{\mathrm{r}}=P_{\text {cell }}$, and $Q_{1}, Q_{\mathrm{r}}$ are initialized, depending, respectively on the class of cells with $R_{1}=$ $R_{\mathrm{r}}=R_{\text {ini }}$ in both vessels. Then it is possible to compute the pressure at the bifurcation point imposed by each of the vessels:

$P_{\mathrm{b}}^{1}=P_{1}+Q_{1} \frac{8 \mu l_{1}}{\pi R_{1}^{4}}$

$P_{\mathrm{b}}^{\mathrm{r}}=P_{\mathrm{r}}+Q_{\mathrm{r}} \frac{8 \mu l_{\mathrm{r}}}{\pi R_{\mathrm{r}}^{4}}$

Computing $P_{\mathrm{b}}^{1}$ and $P_{\mathrm{b}}^{\mathrm{r}}$ with Eqs. (4) and (5) provides different values. To get the same pressure at the bifurcation point, the radius of the vessel with lower pressure at the end is changed. To simplify the notation, let suppose $P_{\mathrm{b}}^{1}>P_{\mathrm{b}}^{\mathrm{r}}$ and then the pressure at the bifurcation point is equal to $P_{\mathrm{b}}=P_{\mathrm{b}}^{1}$. Hence the new radius of the right vessel can be calculated using Eq. (6).

$R_{\mathrm{r}}^{\prime}=\sqrt[4]{Q_{\mathrm{r}} \frac{8 \mu l_{\mathrm{r}}}{\pi\left(P_{\mathrm{b}}-P_{\mathrm{r}}\right)}}$

After changing the radius in one of the vessels, the radius $\left(R_{\mathrm{r}}^{\prime}\right)$ of the bifurcation vessel can be computed using the bifurcation law and, then, the pressure at the entry of the bifurcation vessel can be deduced:

$P_{\mathrm{b}}^{\text {entry }}=P_{\mathrm{b}}+\left(Q_{1}+Q_{\mathrm{r}}\right) \frac{8 \mu l_{\mathrm{b}}}{\pi\left(R_{\mathrm{b}}^{\prime}\right)^{4}}$.

This way, the optimized bifurcation is obtained.

\subsection{A bifurcation with two sub-trees}

Let us consider the situation where two subtrees connected in a bifurcation have to be optimized. Like in the previous case, pressures are calculated at the bifurcation point imposed by the left sub-tree, $P_{\mathrm{b}}^{1}$, and by the right one, $P_{\mathrm{b}}^{\mathrm{r}}$ (we assume that $P_{\mathrm{b}}^{1}>P_{\mathrm{b}}^{\mathrm{r}}$ ). In [3] the pressure at the bifurcation is then averaged based on the blood flow Eq. (8).

$P_{\mathrm{b}}=\frac{P_{\mathrm{b}}^{1} Q_{1}+P_{\mathrm{b}}^{\mathrm{r}} Q_{\mathrm{r}}}{Q_{1}+Q_{\mathrm{r}}}$

Another possibility is to increase/decrease the pressure in one of the sub-trees (like the case with two cells). For example, one can decide to choose $P_{\mathrm{b}}=P_{\mathrm{b}}^{\mathrm{l}}$ and increase the pressure in the right sub-tree. This operation is relatively easy to perform, because the sub-tree is optimized. It only needs to multiply all the radii in the sub-tree by a same coefficient [3] computed by using the pressure $P_{\text {cell }}$ at the extremities. Then, new radii of vessels of the right sub-tree are obtained by the following transformation:

$R_{\mathrm{r}}^{\prime}=R_{\mathrm{r}} \sqrt[4]{\frac{P_{\mathrm{r}}^{\text {end }}-P_{\text {cell }}}{P_{\mathrm{b}}^{\text {end }}-P_{\text {cell }}}}$.

When such a transformation is applied, the corresponding pressures are adjusted. Then, the radius of the bifurcation vessel and the pressure at the end of that vessel can be computed. By applying the described procedure, the optimized subtree starting at the bifurcation point is obtained.

\subsection{Ensuring the given pressure at the entry of the tree}

Using the procedures described above, the optimization of the tree is carried out starting from the extremities and proceeding up to the root. At the end, the optimized tree with the entry pressure depending on the initial radius $\left(R_{\mathrm{ini}}\right)$ is obtained. But to get the fully optimized vascular tree, one needs to get the exact entry pressure given as one of the model parameters.

In the previous subsection, it has been shown how to fit the pressure in the sub-tree when it is optimized. In fact it is enough to apply the aforementioned procedure (multiplying all the radii) to the root node of the tree, using this preset entry pressure. 


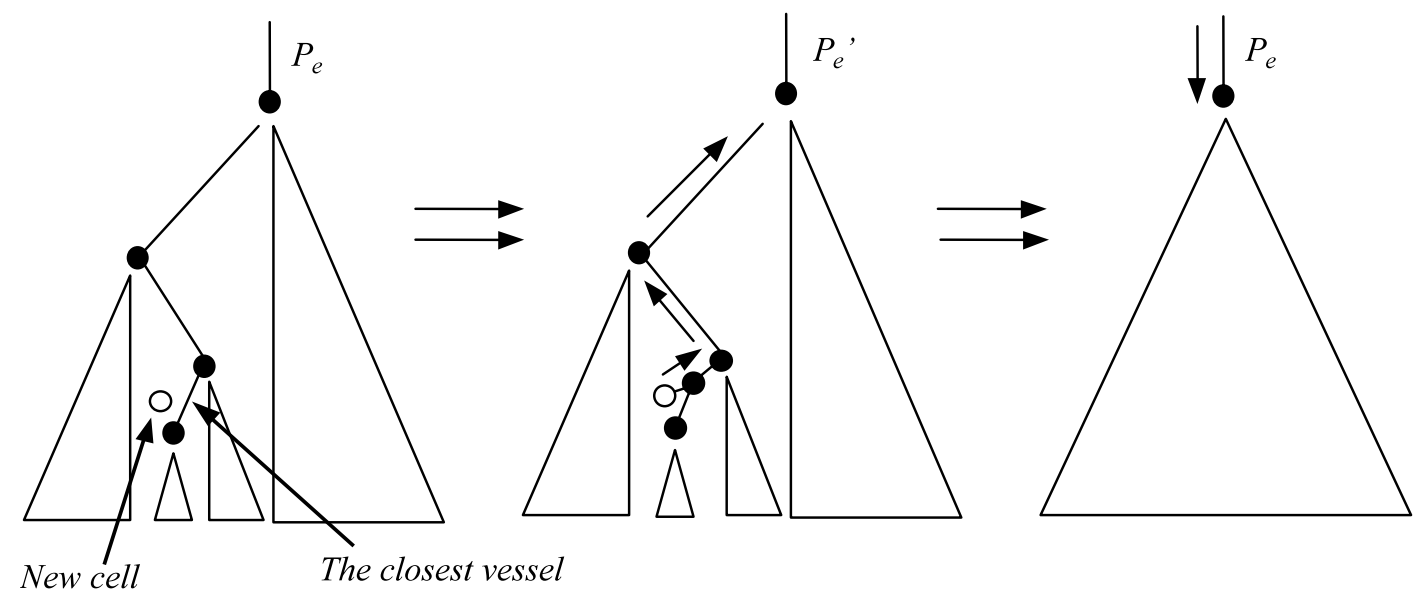

a

b

c

Fig. 2. Optimization by updating. (a) Triangles represent sub-trees. A new bifurcation is added to a fully optimized tree. (b) Pressure is computed at each node along the path from the bifurcation point up to the root, given $P_{\mathrm{e}}^{\prime}$ at the root. (c) Then all radii of the tree are changed to assure the $P_{\mathrm{e}}$ value of pressure given as one of the model parameter.

\subsection{Updating optimization versus global optimization}

When the fully optimized tree is available, it is possible to add/delete a macro-cell to/from the tree (Fig. 2a) and to update it only locally. After adding the new (optimized) bifurcation to the tree, the path can be tracked from the bifurcation point up to the root, and the pressure can be adjusted at each node, using the described above method (Fig. $2 b)$. At the root of the tree, the same procedure as used during the global optimization is applied to ensure the right entry pressure (Fig. 2c). We called this method optimization by updating.

\subsection{Is it possible to speed up the updating algorithm?}

The weak point of the updating algorithm is that in some sub-trees, the pressure and the radius are modified many times. Some nodes of the vascular tree are visited repeatedly to perform several operations. Multiplying coefficients by each other and then using the result to adjust the radius and the pressure can be performed in the same task. Hence instead of 'multiplying' all the nodes in the sub-tree by the coefficient during the fitting phase, it is stored at the root node of this sub-tree. And when the last step is carried out on the whole tree, this available information at the nodes is jointly processed. This algorithm is referred to as 'fast updating'.

\section{Application to the liver arterial tree}

The presented model was implemented in $\mathrm{C}++$ using Microsoft Visual $\mathrm{C}++6.0$. In order to exemplify the whole procedure a hepatic arterial tree is simulated (Fig. 3). Table 1 summarizes the main parameters used to initialize the model.

The 3-D bounding shape of the liver is reconstructed from CT-scan images (Siemens Somaton, 120 slices with $1 \mathrm{~mm}$ thickness). Then, in order to simulate the organ growth, mathematical morphology operations (erosion/dilation) combined with shifting are applied to the shape.

During the first trials, the tree was simulated without restricting the number of candidate vessels and several statistics regarding the perfusion process were collected. In $96 \%$ of the cases, the minimal volume position was obtained using only the five closest vessels. Hence, in order to elimi- 

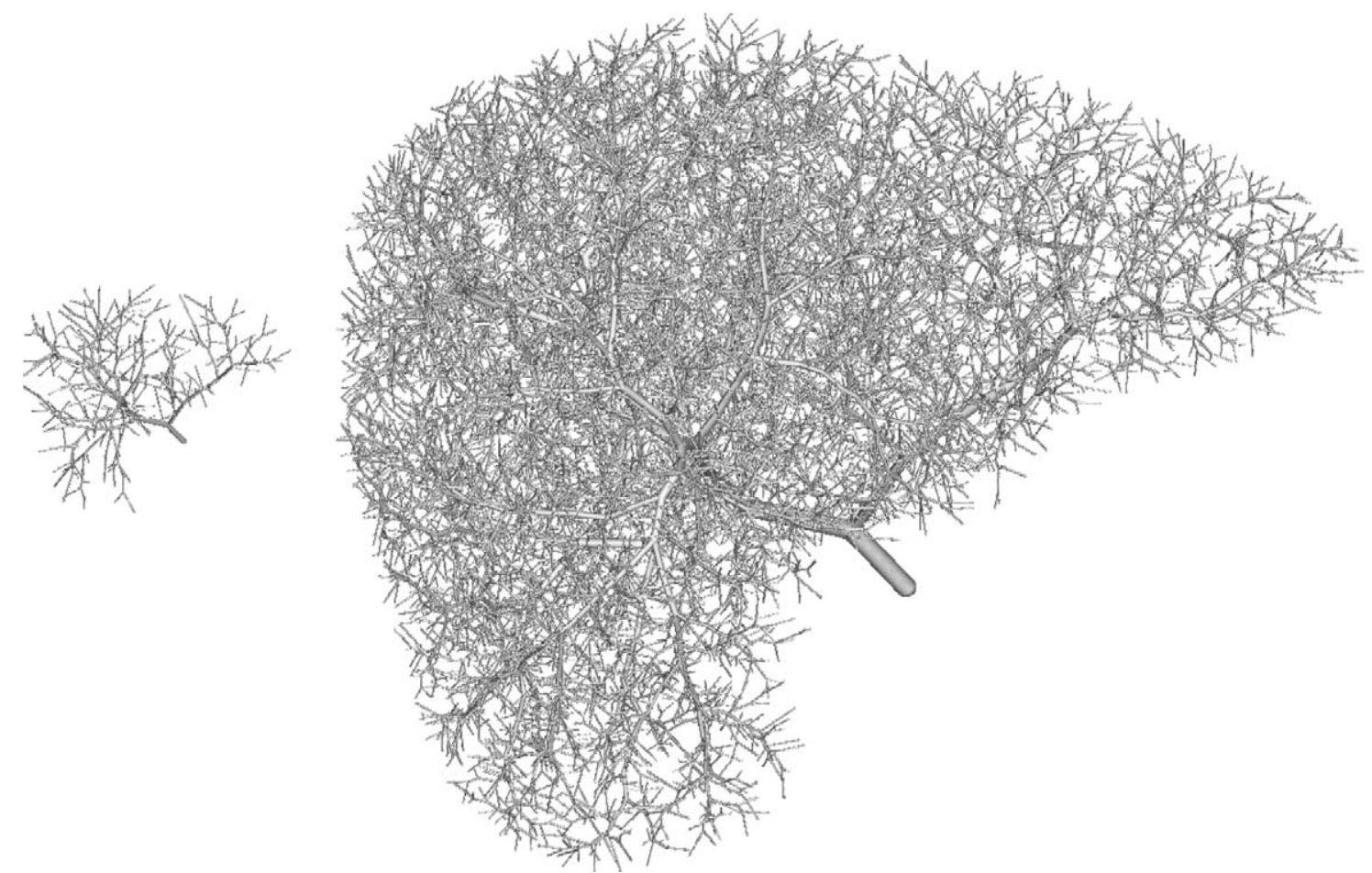

Fig. 3. Various stages of the development of the liver arterial tree model (after the first growth cycle and after the last one).

nate unnecessary computations, the number of candidate vessels is now limited to five.

To validate the proposed improvements in the optimization of vascular trees, the same conditions and parameters set is applied to generate a structurally-equivalent tree (25163 nodes or, equivalently, 12582 macro-cells). Table 2 gives the total execution time for the different optimization methods. The whole tree is built in about $3 \mathrm{~h}$ (using a PC-type computer, PIII $600 \mathrm{MHz}$ ) when the global optimization after each perfusion of a macro-cell is used. When the updating strategy is used this time is reduced by $40 \%$ and the fast updating procedure leads to a three times faster optimization. In comparison, in [4] the computing time for a tree with 4000 macro-cells is approximately $10 \mathrm{~h}$ on a DEC-Alpha $21164 / 333 \mathrm{MHz}$, to get similar vascular trees. The main concepts of the model presented in [3] and the extension described here are close to those reported in [4]. The vessels (rigid tubes forming a binary tree) grow to irrigate macro-cells distributed into a 3-D organ (static shape in [4], growing in [3,5]). In both cases, blood is considered as a Newtonian fluid whose flow is governed by the Poiseuille's law. Similarly, two optimization levels are distinguished: a geometrical optimization of the new bifurcation (minimizing the volume of blood added), and a more global process consisting in re-computing (in slightly different ways) radii of the tree after addition of a new cell. The advances presented in [4] deal with the extension of the 2-D

Table 1

Main model parameters used in the simulation of the hepatic arterial tree

\begin{tabular}{ll}
\hline Number of growth shape cycles & 15 \\
Volume growth $\left(\mathrm{cm}^{3}\right)$ & $88 \rightarrow 1500$ \\
Number of macro-cells & 12000 \\
Hepatic artery pressure $(\mathrm{mmHg})$ & 98 \\
Blood pressure at the macro-cell $(\mathrm{mmHg})$ & 50 \\
Blood flow at the macro-cell $(\mathrm{ml} / \mathrm{min})$ & 0.033 \\
$\gamma$ parameter of the bifurcation law & 2.7 \\
\hline
\end{tabular}


Table 2

Comparison of the total simulation times with the proposed methods

\begin{tabular}{llc}
\hline Optimization method & Time (min) & $\%$ \\
\hline Global & 187 & 100 \\
'Updating' & 107.5 & 57.5 \\
'Fast updating' & 62.6 & 33.5 \\
\hline
\end{tabular}

model first presented in [2], and the introduction of terminal flow variability which influences the structure and the geometry of the vascular tree. This property could be compared to the one we used in [3], where several classes of cells, with different geometrical and physiological properties coexist in a same organ, leading to various kinds of vascular regions (hyper-vascularized/ normal for example).

Although the model relies on simplified properties, its branching patterns and morphometric features observed through visual rendering as well as slice CT-scanner simulations and texture measurements are consistent with in-vitro and in-vivo experiments [12]. As far as future works are concerned, several physiologically-based improvements can be expected by taking into account the blood flow distribution heterogeneity (possibly related to specific metabolic needs [13]), shear stress participating to the growth process [14], microcirculation mechanisms for the remodeling or revascularization of the networks. Here one can notice that additional anatomical and physiological data are required, but they are more difficult to collect than data measured on other vascular system like coronary trees $[15,16]$.

\section{Conclusions and future research}

The present algorithm provides better computational performances for the simulation of arterial tree growth in three dimensions. It can be used for preliminary studies aimed at evaluating the impact of variable or parameter changes on a statistical basis.
In medical practice, the most common way to observe and characterize the vascular network modifications is to acquire images (CT scan, MRI, and Ultrasound...). Vascular trees simulated by the model can be used to form physiologically sound images as it has been shown in [12].

Apart from the many improvements that can be introduced into the model (e.g. anostomoses, elasticity of the vessel walls, pulsative feature of the blood flow ...), various enhancements of the above algorithm are in progress including more realistic shape constraints, improved boundary conditions, multiple connected trees (e.g. arterial and venous). More effective algorithms to detect potential intersections and crossings between vessels during the growth process have to be designed along with the adaptive corrections.

\section{References}

[1] V. Meier, Realistic visualization of abdominal organs and its application in laparoscopic surgery simulation, $\mathrm{PhD}$ Swiss Fed Inst Technol Zurich, 1999.

[2] W. Schreiner, F. Buxbaum, Computer optimization of vascular trees, IEEE Transactions on Biomedical Engineering 40 (5) (1993) 482-491.

[3] J. Bézy-Wendling, A. Bruno, A 3-D dynamic model of vascular trees, Journal of Biological Systems 7 (1) (1999) $11-31$

[4] R. Karch, F. Neumann, M. Neumann, W. Schreiner, A three-dimensional model for arterial tree representation, generated by constrained constructive optimization, Computers in Biology and Medicine 29 (1999) 19-38.

[5] R. Karch, F. Neumann, M. Neumann, W. Schreiner, Staged growth of optimized arterial model trees, Annals of Biomedical Engineering 28 (2000) 495-511.

[6] Y. Rolland, J. Bézy-Wendling, R. Duvauferrier, A. Bruno, Modeling of the parenchymous vascularization and perfusion, Investigative Radiology 34 (3) (1999) 171 175.

[7] A. Kamiya, T. Togawa, Optimal branching structure of the vascular trees, Bulletin of Mathematical Biophysics 34 (1972) 431-438.

[8] D.L. Cohn, Optimal systems: I. The vascular system, Bulletin of Mathematical Biophysics 16 (1954) 59-74.

[9] C.D. Murray, The physiological principle of minimum work applied to the angle of branching arteries, Journal of General Physiology 9 (1926) 835-841.

[10] B. Mandelbrot, The Fractal Geometry of Nature, W.H. Freeman and Company, New York, 1977.

[11] M. Zamir, Optimality principles in arterial branching, 
Journal of Theoretical Biology 62 (1976) 227-251.

[12] Y. Rolland, J. Bézy-Wendling, R. Duvauferrier, J.L. Coatrieux, Slice simulation from a model of the parenchymous vascularization to evaluate texture features, Investigative Radiology 34 (3) (1999) 181-184.

[13] J.B. Bassingthwaigthe, R.B. King, S.A. Roger, Fractal nature of regional myocardial blood flow heterogeneity, Circulation Research 65 (3) (1989) 578-590 September.

[14] W.J. Hacking, E. VanBavel, J.A.E. Spaan, Shear stress is not sufficient to control growth of vascular networks: a model study, American Journal of Physiology 270 (1995) H364-H375 July.

[15] G.S. Kassab, C.A. Rider, N.J. Tang, Y.C. Fung, Morphometry of pig coronary arterial trees, American Journal of Physiology 265 (1993) H350-H365 July.

[16] R.J. Tomanek, P.J. Palmer, G.L. Peiffer, K.L. Schreiber, C.L. Eastham, M.L. Marcus, Morphometry of canine coronary arteries, arterioles, and capillaries during hypertension and left ventricular hypertrophy, Circulation Research 58 (1986) 38-46. 